\title{
A flexible format for exchanging pulsar data
}

\author{
D.R. Lorimer ${ }^{1}$, A. Jessner ${ }^{1}$, J.H. Seiradakis ${ }^{2}$, \\ K.M. Xilouris ${ }^{5}$, M. Kramer ${ }^{1}$, and R. Wielebinski ${ }^{1}$ \\ A.G. Lyne ${ }^{3}, \quad$ N. D’Amico ${ }^{4}$, \\ A. Athanasopoulos ${ }^{2}$, \\ 1 Max-Planck-Institut für Radioastronomie, Auf dem Hügel 69, D-53121 Bonn, Germany \\ 2 University of Thessaloniki, Department of Physics, Section of Astrophysics, Astronomy and Mechanics, GR-54006, Greece \\ ${ }^{3}$ University of Manchester, Nuffield Radio Astronomy Laboratories, Jodrell Bank, Macclesfield, Cheshire, SK11 9DL, UK \\ ${ }^{4}$ Osservatorio Astronomico di Bologna, via Zamboni 33, 40126 Bologna, Italy \\ 5 National Astronomy and Ionospheric Center, Arecibo Observatory, P.O. Box 995, Arecibo, Puerto Rico 00613
}

Received June 6; accepted August 28, 1997

\begin{abstract}
We describe a data format currently in use amongst European institutions for exchanging and archiving pulsar data. The format is designed to be as flexible as possible with regard to present and future compatibility with different operating systems. One application of the common format is simultaneous multi-frequency observations of single pulses. A data archive containing over 2500 pulse profiles stored in this format is now available via the Internet, together with a small suite of computer programs that can read, write and display the data.
\end{abstract}

Key words: pulsars: general — astronomical databases: miscellaneous

\section{Introduction}

The European Pulsar Network ("EPN") is an association of European astrophysical research institutes that co-operate in the subject of pulsar research. All institutes have up until now developed their own individual hardware and software facilities tailored to their own requirements and will, of course, continue to do so in future. Contact and co-operation has always existed between the scientists of the member institutes and outside, but the lack of a common standard format for pulsar data has hampered previous collaborative research efforts.

In this paper, we describe a flexible format that we have developed for exchanging data between EPN pulsar groups. The format has some generic similarities to the widely used FITS format (Wells et al. 1981) but has been designed to meet the specific needs of the EPN. The format has proved so successful that we now advocate its use as a useful world-wide utility for pulsar data exchange. To

Send offprint requests to: D.R. Lorimer

e-mail: dunc@mpifr-bonn.mpg.de aid implementation of the format, we have written a suite of freely available Fortran- 77 sub-routines which can be easily incorporated within existing software to read and write data in this format. Astrophysical applications of such a format currently being pursued by EPN groups include the establishment of a data bank of pulse profiles as well as simultaneous observations of pulsars by several European observatories.

\section{The EPN format}

The underlying principles of the format result from a number of requirements. This was essentially a balance between the need for efficient data storage and providing sufficient information about the data for potential users. Specifically, the following requirements had to be met:

- Operating system independence: To make the data format as portable as possible between present and future operating systems, we have opted to use only ASCII-data throughout. We have arrange these data so that words are aligned over 80-byte boundaries, this simplifies inspection and printing of the files.

- Completeness: The data should contain all information for the identification of the source and the observing circumstances useful for further analyses of the data by others.

- Compactness: Descriptive information should not dominate the format. The measured values that form the bulk of a block of data are given as scaled fourcharacter hexadecimal numbers, giving a dynamic range of up to $65536: 1$.

- Versatility: The format should be suitable for continuously sampled multi-channel filterbank search data, synchronous integrated and single-pulse data as well as processed data. In addition, we have designed the format, so that it can be used for observations of pulsars outside the radio regime i.e. variable units for the observing frequency and bandwidth, as well as 
topocentric telescope coordinates which are time variable for satellite observatories. Space is left for more descriptors, future adaptations and expansions.

- Simplicity and ease of access: We describe a data format consisting of a standardised fixed-length header with a variable length data structure attached to it. The header fully describes the structure of the data, which is not changed within one file but can vary between files. In this way, it is possible to calculate the length of a data block within each file after reading its header. The file can then be opened for random access with fixed block length, faster than a sequential read.

Many of the above mentioned requirements were already met by a format in use at Jodrell Bank to which we made suitable modifications and extensions to make it more flexible.

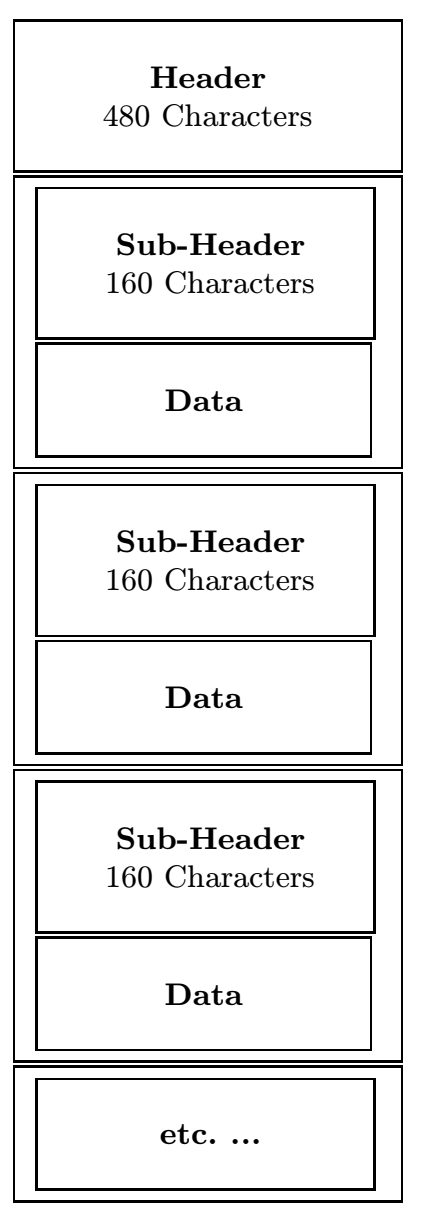

Fig. 1. Schematic representation of an EPN data block

Each EPN file consists of one or more EPN blocks. The basic structure of an EPN block is shown in Fig. 1. Each file has a common fixed length header followed by a number of individual data streams of equal length. The header describes the data, containing information on the pulsar itself, the observing system used to make the observation as well as some free-form information about the processing history of the data. The onus is on the site-specific conversion process to ensure correct conversion to the standardised entries and reference to common catalogues (e.g. the Taylor et al. 1993, catalogue of pulsar parameters). The full list of header variables is given in Tables 1 and 2 .

The data streams themselves may be outputs of different polarisation channels, or individual channels (bands) of a filterbank or a combination thereof. In total, there may be $N_{\text {freq }}$ data streams of i.e. different frequencies for each polarisation. Each data stream starts with a small, fixed length sub-header in front of the actual data values. The number of data streams and their length may vary between different EPN files, but is constant within each file. A character field and an ordinal number is provided for each stream for its identification.

\section{Simultaneous observations of single pulses}

Pulsars are, in general, very weak sources, typically requiring the addition of several thousand individual pulses with a large radio telescope equipped with sensitive receivers in order to attain a sufficiently large signal-to-noise ratio. The brightest pulsars are, however, strong enough so that individual pulses can be observed. These pulses are known to exhibit great variety in morphology and polarimetric properties from one pulse to the next (see for example Lyne \& Smith 1990). It is presently unclear whether the same features in the individual pulses are present at different observing frequencies. One of the current research topics being carried out by EPN is a multi-frequency study of single pulses. The project requires the pulses observed at different telescopes to be time-aligned and thus the format described above has an ideal application in this project. After conversion of the data into this format, the time alignment of the pulses and subsequent statistical analyses is a relatively straight-forward procedure. As an example, a set of pulses from PSR B0329+54 observed simultaneously at Bologna (410 MHz), Jodrell Bank (1.404 GHz) and Effelsberg $(4.850 \mathrm{GHz})$ are shown in Fig. 2. The pulses show a remarkable similarity at these three frequencies, although counter examples are also observed. Full results of this study will be published shortly.

\section{The EPN pulse profile archive}

As well as being used for data interchange between EPN members, the common format forms the basis of a pulse profile archive presently being maintained at the Max-Planck-Institut für Radioastronomie in Bonn. The idea of the archive is to build up a useful collection of pulse profiles which anybody with access to the Internet can use. Presently, around 2500 pulse profiles are stored in this format. The profiles 
Table 1. A description of the EPN format variables

\begin{tabular}{|c|c|c|c|c|}
\hline Position & Name & Format & Unit & Comment \\
\hline 1 & version & A8 & & EPN + version of format (presently EPN05.00) \\
\hline 9 & counter & $\mathrm{I} 4$ & & No. of records contained in this data block \\
\hline 13 & history & $\mathrm{A} 68$ & & comments and history of the data \\
\hline 81 & jname & A12 & & pulsar jname \\
\hline 93 & name & $\mathrm{A} 12$ & & common name \\
\hline 105 & $P_{\text {bar }}$ & F16.12 & $\mathrm{s}$ & current barycentric period \\
\hline 121 & $\mathrm{DM}$ & F8.3 & $\mathrm{pc} \mathrm{cm}^{-3}$ & dispersion measure \\
\hline 129 & $\mathrm{RM}$ & F10.3 & $\operatorname{rad~} \mathrm{m}^{-2}$ & rotation measure \\
\hline 139 & CATREF & A6 & & pulsar parameter catalogue in use \\
\hline 145 & BIBREF & A 8 & & bibliographical reference key (or observer's name) \\
\hline 153 & & $8 \mathrm{X}$ & & blank space free for future expansion \\
\hline 161 & $\alpha_{2000}$ & $\mathrm{I} 2, \mathrm{I} 2, \mathrm{~F} 6.3$ & hhmmss & right ascension of source \\
\hline 171 & $\delta_{2000}$ & $\mathrm{I} 3, \mathrm{I} 2, \mathrm{~F} 6.3$ & ddmmss & declination of source \\
\hline 182 & telname & A8 & & name of the observing telescope (site) \\
\hline 190 & $\mathrm{EPOCH}$ & F10.3 & day & modified Julian date of observation \\
\hline 200 & OPOS & F8.3 & degrees & position angle of telescope \\
\hline 208 & PAFLAG & A1 & & $\mathrm{A}=$ absolute polarisation position angle, else undefined \\
\hline 209 & TIMFLAG & A1 & & $\mathrm{A}=$ absolute time stamps (UTC), else undefined \\
\hline 210 & & $31 \mathrm{X}$ & & blank space free for future expansion \\
\hline 241 & $x_{\text {tel }}$ & F17.5 & $\mathrm{m}$ & topocentric $X$ rectangular position of telescope \\
\hline 258 & $y_{\text {tel }}$ & F17.5 & $\mathrm{m}$ & topocentric $Y$ rectangular position of telescope \\
\hline 275 & $z_{\text {tel }}$ & F17.5 & $\mathrm{m}$ & topocentric $Z$ rectangular position of telescope \\
\hline 292 & & $29 \mathrm{X}$ & & blank space free for future expansion \\
\hline 321 & CDATE & $\mathrm{I} 2, \mathrm{I} 2, \mathrm{I} 4$ & $\mathrm{~d} \mathrm{~m} \mathrm{y}$ & creation/modification date of the dataset \\
\hline 329 & SCANNO & I4 & & sequence number of the observation \\
\hline 333 & SUBSCAN & $\mathrm{I} 4$ & & sub-sequence number of the observation \\
\hline 337 & $N_{\mathrm{pol}}$ & $\mathrm{I} 2$ & & number of polarisations observed \\
\hline 339 & $N_{\text {freq }}$ & $\mathrm{I} 4$ & & number of frequency bands per polarisation \\
\hline 343 & $N_{\text {bin }}$ & $\mathrm{I} 4$ & & number of phase bins per frequency (1-9999) \\
\hline 347 & $t_{\text {bin }}$ & F12.6 & $\mu \mathrm{s}$ & duration (sampling interval) of a phase bin \\
\hline 359 & $t_{\mathrm{res}}$ & F12.6 & $\mu \mathrm{s}$ & temporal resolution of the data \\
\hline 371 & $N_{\text {int }}$ & I6 & & number of integrated pulses per block of data \\
\hline 377 & $n_{\text {cal }}$ & $\mathrm{I} 4$ & $t_{\mathrm{bin}}$ & bin number for start of calibration signal \\
\hline 381 & $l_{\text {cal }}$ & $\mathrm{I} 4$ & $t_{\text {bin }}$ & length of calibration signal \\
\hline 385 & FLUXFLAG & A1 & & $\mathrm{F}=$ data are flux calibrated in $\mathrm{mJy}$, else undefined \\
\hline 386 & & $15 \mathrm{X}$ & & blank space free for future expansion \\
\hline 401 & & $80 \mathrm{X}$ & & blank space free for future expansion \\
\hline
\end{tabular}

themselves have usually already been, or about to be, published so that full credit for any subsequent use via the database can go to the contributing authors. The archive has the following URL address: http://www.mpifrbonn.mpg.de/pulsar/data/. Authors are encouraged to make their data available to this archive and should contact Duncan Lorimer (e-mail: dunc@mpifr-bonn.mpg.de) if they wish to do this.

Acknowledgements. We thank the referee, Dick Manchester, for useful comments on an earlier version of the manuscript. The EPN was funded under Brussels Human Capital and Mobility grant number CHRX-CT94-0622.

\section{Appendix}

\section{A. Format compatible software}

To incorporate the capability to read and write data in this format within existing analysis software, a simple routine exists which can read and write data in this format. In addition, we have written some sample programs which can plot the data and display the header parameters. The software are written in Fortran - 77 and have been packaged into a single UNIX tar file which is freely available via the Internet. To down-load the package, log into the anonymous ftp area: ftp.mpifr-bonn.mpg.de, with the username anonymous using your complete E-mail address as the password. Once logged in, issue the following commands: 
Table 2. The sub-header variables. ${ }^{*} \Theta(x)$ is the Heaviside-function: $=1$ if $x \geq 0$ and $=0$ elsewhere

\begin{tabular}{|c|c|c|c|c|}
\hline Position & Name & Format & Unit & Comment \\
\hline 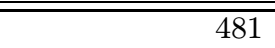 & IDfield & $\overline{\mathrm{A} 8}$ & & 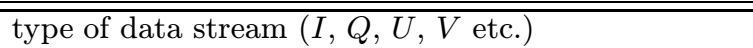 \\
\hline 489 & $n_{\text {band }}$ & I4 & & ordinal number of current stream \\
\hline 493 & $n_{\text {avg }}$ & I4 & & number of streams averaged into the current one \\
\hline 497 & $f_{0}$ & F12.8 & & effective centre sky frequency of this stream \\
\hline 509 & $U_{f}$ & A 8 & & unit of $f_{0}$ \\
\hline 517 & $\Delta f$ & F12.6 & & effective band width \\
\hline 529 & $U_{\Delta}$ & A8 & & unit of $\Delta f$ \\
\hline 537 & $t_{\text {start }}$ & F17.5 & $\mu \mathrm{s}$ & time of first phase bin wrt EPOCH \\
\hline 554 & & $7 \mathrm{X}$ & & blank space free for future expansion \\
\hline 561 & SCALE & E12.6 & & scale factor for the data \\
\hline 573 & OFFSET & E12.6 & & offset to be added to the data \\
\hline 585 & RMS & E12.6 & & rms for this data stream \\
\hline 597 & $P_{\text {app }}$ & F16.12 & $\mathrm{s}$ & apparent period at time of first phase bin \\
\hline 613 & & $28 \mathrm{X}$ & & blank space free for future expansion \\
\hline 641 & $\operatorname{Data}(1)$ & $\mathrm{I} 4$ & & scaled data for first bin \\
\hline $4\left(N_{\text {bin }}-1\right)+641$ & $\operatorname{Data}\left(N_{\text {bin }}\right)$ & I4 & & data for last bin of stream \\
\hline $640+N_{\text {records }} * 80$ & & & & $\begin{array}{l}\text { end of first stream, } \\
N_{\text {records }}=\operatorname{INT}\left(N_{\text {bin }} \cdot 0.05\right)+\Theta\left(\left(4 N_{\text {bin }} \bmod 80\right)-1\right)^{*}\end{array}$ \\
\hline
\end{tabular}
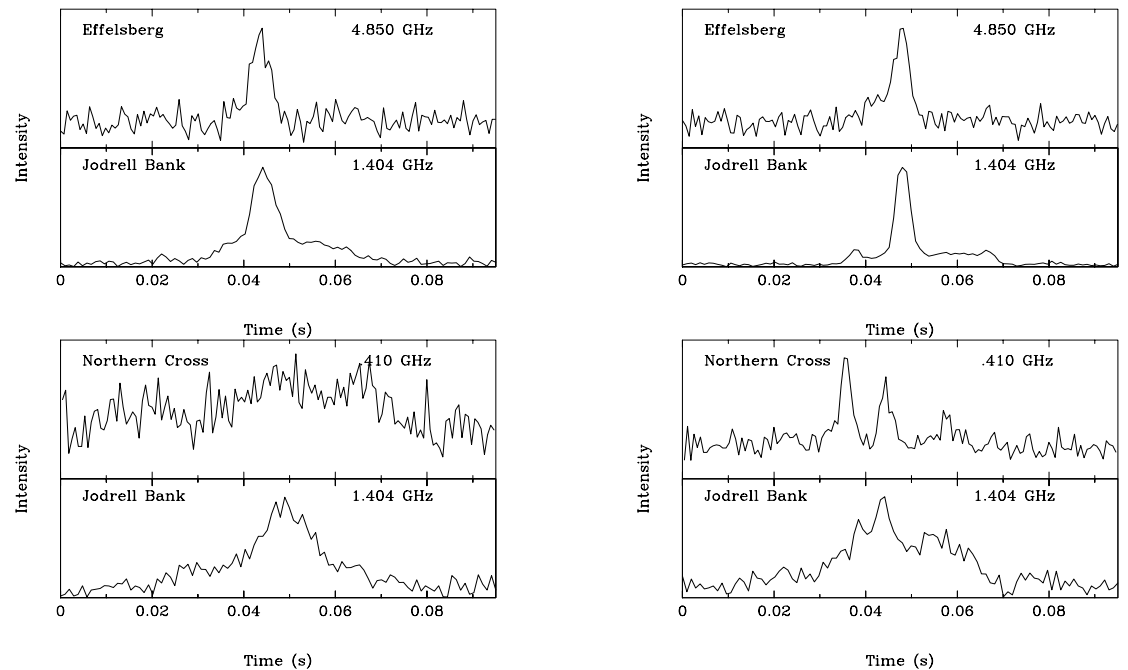
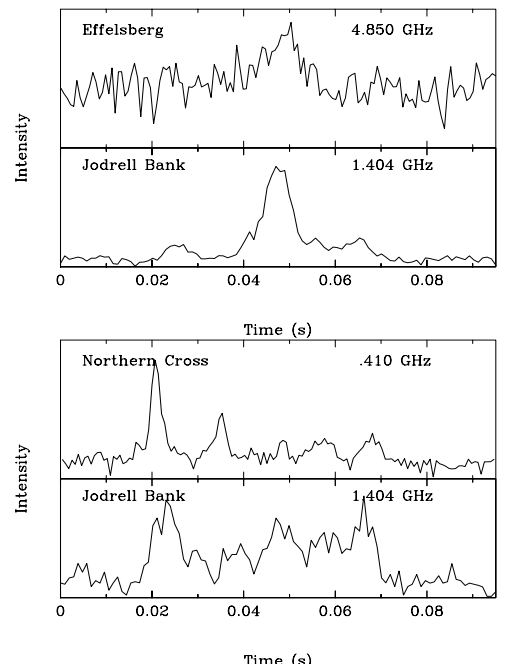

Fig. 2. Time-aligned single pulses for PSR B0329+54 observed simultaneously at Effelsberg (4.850 GHz) and Jodrell Bank $(1.404 \mathrm{GHz})$ shown in the upper panel and at Bologna $(410 \mathrm{MHz})$ and Jodrell Bank $(1.404 \mathrm{GHz})$ in the lower panel. The data were processed using the EPN format

cd pub/pulsar

binary

get epnsoft.tar.gz

Alternatively, the file can be down-loaded from the EPN Internet home-page:

http://www.mpifr-bonn.mpg.de/pulsar/epn/.

To uncompress and extract the contents of the tar file on a UNIX operating system, issue the commands:

gunzip epnsoft.tar.gz

tar xvf epnsoft.tar
The present package contains some sample data and two example programs - "plotepn" and "viewepn" which plot and view EPN files respectively. The ASCII file O0README in this packages gives further details of the software and how to use it.

\section{References}

Lyne A.G., Smith F.G., 1990, Pulsar Astronomy. Cambridge University Press

Taylor J.H., Manchester R.N., Lyne A.G., 1993, ApJS 88, 529 Wells D.C., Greisen E.W., Harten R.H., 1981, A\&AS 44, 363 